\title{
Inscripciones sobre vidrio. Una nueva marca griega sobre fondo de botella hallada en la ciudad romana de Los Bañales de Uncastillo (Zaragoza, España)
}

\section{Inscriptions on glass. A new Greek mark on a glass bottle discovered in the Roman city of Los Bañales de Uncastillo (Zaragoza, Spain)}

\section{Javier Andreu Pintado}

Universidad de Navarra. Edificio Ismael Sánchez Bella. Departamento de Historia, Historia del Arte y Geografía Ctra. Río Sadar, s/n, E-31009 Pamplona (Navarra)

jandreup@unav.es

\section{Aitor Blanco-PÉrez}

Universidad de Navarra. Edificio Ismael Sánchez Bella. Departamento de Filología

Ctra. Río Sadar, s/n, E-31009 Pamplona (Navarra)

ablancop@unav.es

Aquest treball dona a conèixer una singular marca en grec sobre un fragment de vidre, pertanyent segurament al fons d'una ampolla, recuperat a les excavacions arqueològiques en curs a la ciutat romana de Los Bañales, a la vall de l'Ebre. Després de revisar i ordenar els usos del vidre com a suport epigràfic al món romà, es relaciona el text amb el possible nom d'algun dels uitrarii testificats a la Mediterrània i es proposa, també, amb criteris paleogràfics i arqueològics, una datació per al document. 
This paper presents a very singular Greek mark on a piece of glass, probably from the base of a bottle, found as a result of the archaeological excavations currently underway in the Roman city of Los Bañales, in the Ebro valley (Spain). After a general review of the application and purpose of Roman epigraphy in the case of glass vessels, it is argued that the inscribed mark probably refers to the name of one of the uitrarii attested in the Mediterranean. On the basis of palaeographical and archaeological criteria, a chronology for the piece is also offered.

\section{KEYWORDS}

GREEK EPIGRAPHY, EPIGRAPHY ON GLASS, ROMAN GLASS, ROMAN TRADE, LOS BAÑALES DE UNCASTILLO

El presente trabajo da a conocer una singular marca en griego sobre un fragmento de vidrio, perteneciente seguramente al fondo de una botella, recuperado en las excavaciones arqueológicas en curso en la ciudad romana de Los Bañales, en el valle del Ebro. Tras revisar y ordenar los usos del vidrio como soporte epigráfico en el mundo romano, se relaciona el texto con el posible nombre de alguno de los uitrarii atestiguados en el Mediterráneo y se propone, también, con criterios paleográficos y arqueológicos, una datación para el documento.

\section{PALABRAS CLAVE}

EPIGRAFÍA GRIEGA, EPIGRAFÍA SOBRE VIDRIO, VIDRIO ROMANO, COMERCIO ROMANO, LOS BAÑALES DE UNCASTILLO

En la última década, la investigación arqueológica ha puesto en el mapa la ciudad romana de Los Bañales de Uncastillo (Uncastillo, Zaragoza), ubicada en la parte septentrional del conuentus Caesaraugustanus, dentro de la Tarraconense hispana, en la parte norte del valle medio del Ebro (Andreu, 2011). Su proceso de monumentalización —iniciado en el siglo I a. C. en función de la posición de la ciudad al pie de la vía Caesar Augusta-Beneharnum y abruptamente interrumpido a finales del siglo II d. C. (Andreu y Delage, 2017, con bibliografía) - la singularidad y monumentalidad de su foro (Romero, 2017), el aparato escultórico y epigráfico del mismo (Romero y Andreu, 2018, así como, para el repertorio epigráfico, Andreu, 2016) y, en definitiva, la continuidad en las excavaciones arqueológicas desde 2009 han convertido el lugar en uno de los enclaves más dinámicos en la arqueología hispanorromana o, al menos, del norte peninsular (fig. 1). Además, para los estudiosos del vidrio, Los Bañales de Uncastillo, en cualquier caso, ya ocupaba un lugar importante en la bibliografía especializada por haber sido el primer enclave de la península ibérica en el que se documentasen ventanas hemiesféricas en vidrio soplado, a partir de un ejemplar procedente de las termas de la ciudad y que se conserva en el Museo de Zaragoza (fig. 2) (Ortiz y Paz, 1998: 443 y Ortiz y Paz, 2011).

En el transcurso de la campaña de excavaciones del verano de 2019, centrada en una monumental domus altoimperial ubicada al sur de uno de los grandes decumani y al oeste de uno de los cardines de la ciudad (Peñalver y Delage, 2018: 235-239 y Uribe, 2015: 340-343), en el barrio septentrional de la misma (fig. 3), se produjo el hallazgo de un 
Figura 1. Situación de la ciudad romana de Los Bañales de Uncastillo (mapa: Óscar Ribote).
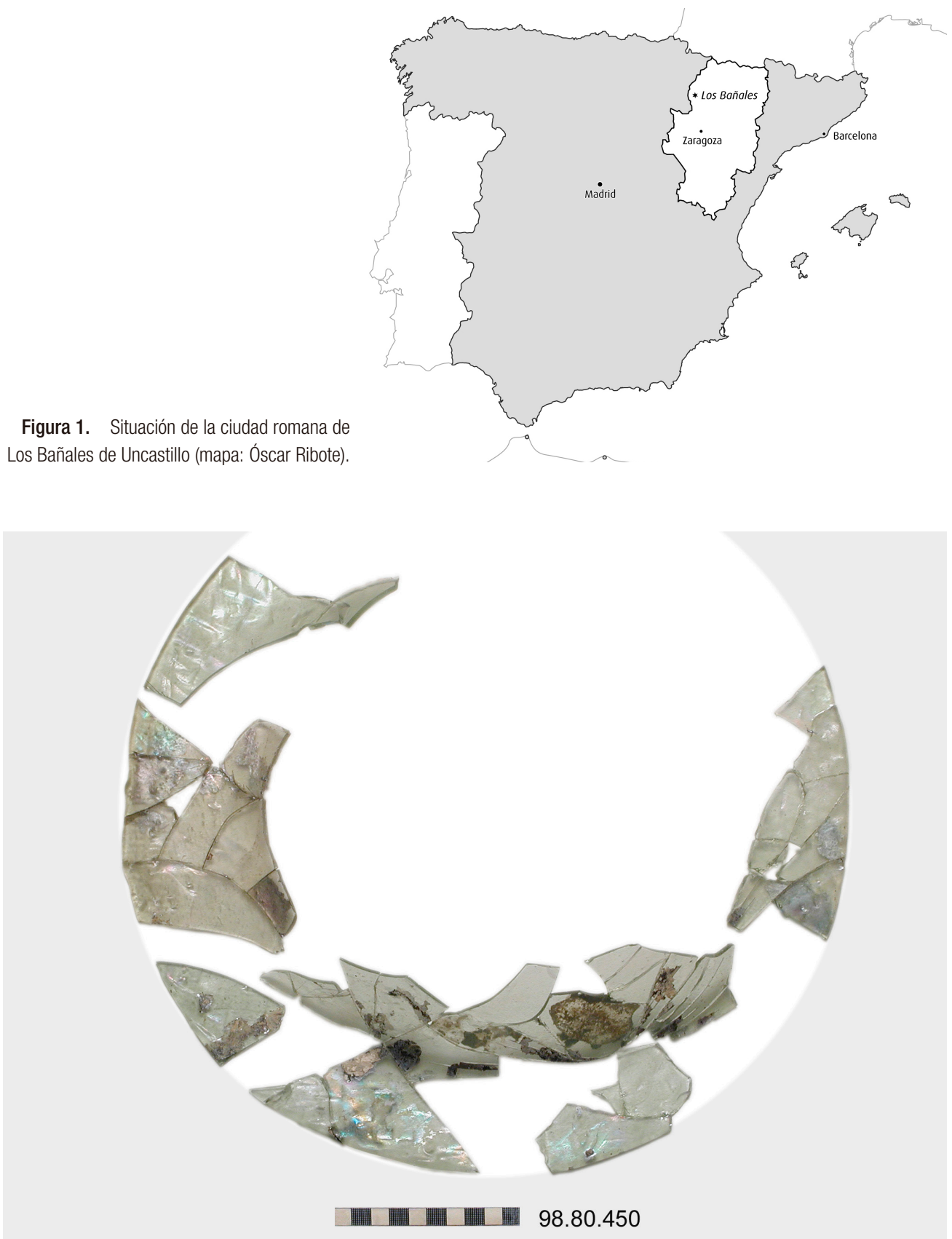

Figura 2. Montaje fotográfico de los fragmentos de ventana circular descubiertos por José M. ${ }^{a}$ Viladés en el sector oeste de las termas públicas de Los Bañales de Uncastillo (Foto: Esperanza Ortiz y Juan Á. Paz). 


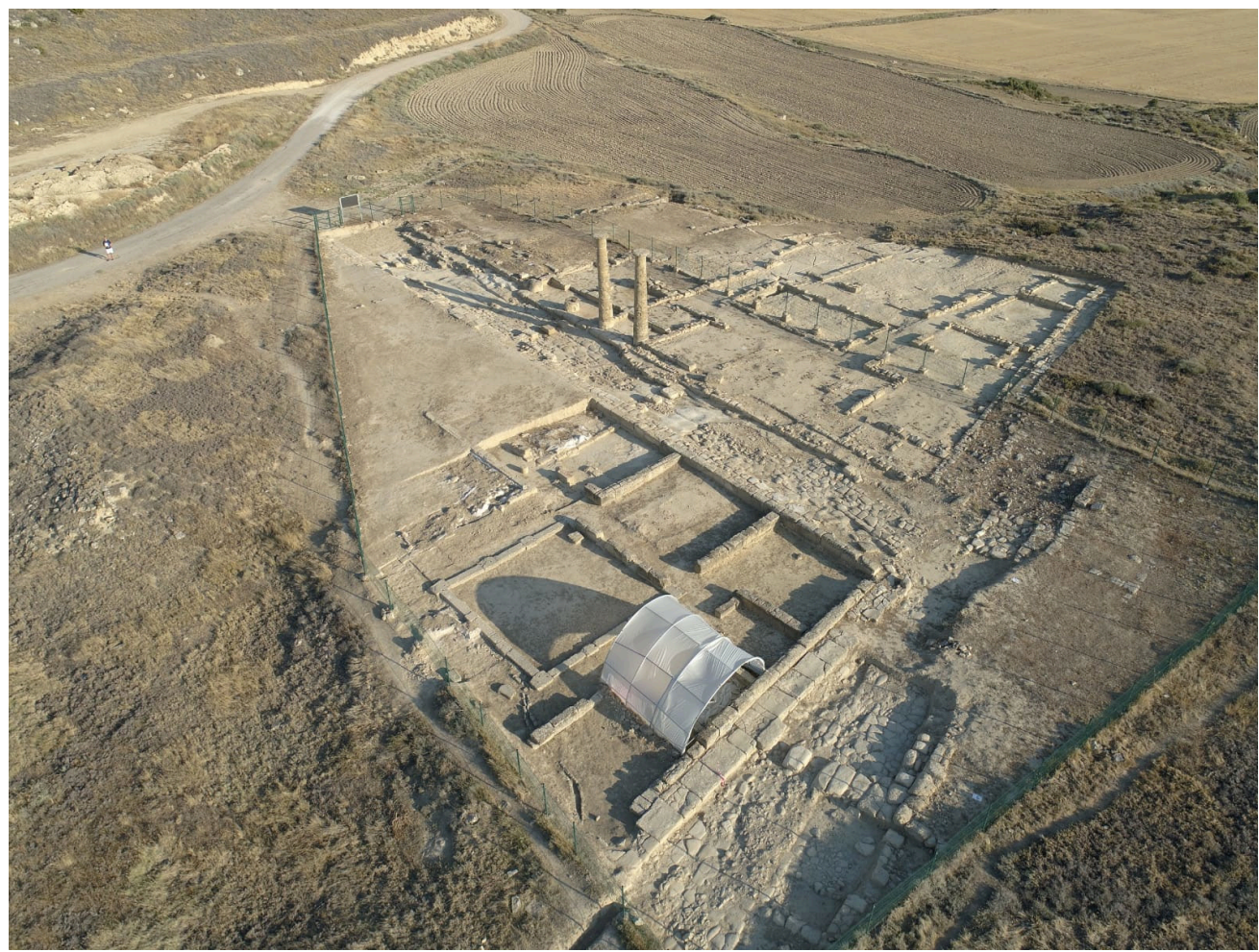

Figura 3. Vista cenital del barrio septentrional de la ciudad romana de Los Bañales de Uncastillo (Foto: Juanmi Círez).

fragmento de vidrio con una marca textual en molde. Éste apareció con la característica pátina verdosa y escamada típica de los procesos de corrosión que afectan a los vidrios antiguos y, en particular, a los romanos. Tras su minuciosa limpieza y restauración (fig. 4), el fragmento ha resultado ser de pátina clara, traslúcido, de muy buena factura, compacto y $\sin$ apenas burbujas de gas. Mide $2,4 \mathrm{~cm}$ de largo y 1,2 $\mathrm{cm}$ de alto, con un espesor de $0,9 \mathrm{~cm}$ y con las letras capitales griegas HTIW — que, como veremos, muy probablemente debamos leer al revés-, de 0,5 cm de altura y entre 0,5 y 0,2 cm de ancho, en relieve y realizadas a molde, como era habitual en las producciones de vidrio soplado. La pieza, en concreto, procede del nivel de abandono del cardo oriental, donde, sobre el enlosado del citado cardo, fue hallada con materiales muy variados — sobre todo cerámica y elementos constructivos romanos del tipo tegulae o imbrices - datados los más tardíos en torno a la segunda mitad del siglo II d. C. En esta fecha se iniciaría una crisis urbana local de notable alcance, como evidencia el descuido del callejero de la ciudad (Andreu et al., 2020), que, desde una perspectiva epigráfica, parece convenir también con la datación del recipiente del que este fragmento formó parte, como veremos a continuación. 


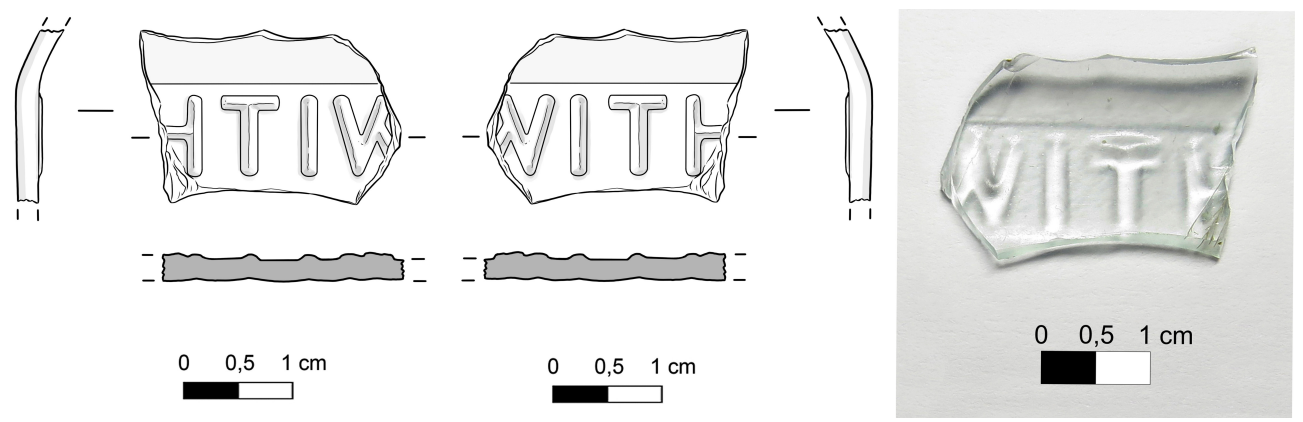

Figura 4. Dibujos y fotografía del fragmento de vidrio con inscripción en griego para lectura retrógrada (Iñaki Diéguez). 4. 1. y 4. 2.: Dibujos. 4. 3.: Fotografía (Iñaki Diéguez).

Este tipo de marcas inscritas — habitualmente valoradas en la investigación sobre el vidrio antiguo (Foy y Nenna, 2006 y Nenna, 2001: 328-329, n. ${ }^{\text {os }}$ 141-145, o Diani y Mandruzzato, 2012: 9-110) ya desde los trabajos generales de referencia a comienzos y mediados del siglo xx (Kisa, 1908: 848-867 o Vigil, 1969: 90-93 y 112)— no han gozado, todavía, del predicamento que merecen en la investigación epigráfica tradicional, al margen de su modélica inclusión en uno de los fascículos sobre instrumenta domestica de la serie The Roman Inscriptions of Britain (Collingwood y Wright, 1991: 86-127) y su ocasional -y casi siempre parca- consideración en el marco de la denominada epigrafia della produzione e della distribuzione (Sternini, 1994), también calificada acertadamente como inscribed economy (Sternini, 1993). Sí se alude a los "sellos de vidrio" en algunos manuales de referencia (Ozcáriz, 2009: 542-543), mientras que otros repertorios y volúmenes más difundidos incluyen este tipo de inscripciones entre la miscelánea categoría de los «textos de producción, distribución o propiedad» (Cooley, 2012: 82-104 y 108-111, donde sobre todo se atiende como ejemplo a las botellas de Puteoli y Baiae, ya estudiadas por Picard, 1959, por Painter, 1975 y por Ostrow, 1979). En otras ocasiones se omiten las inscripciones sobre vidrio, incluso cuando se alude a una categoría tan específica como la del instrumentum inscriptum (Keppie, 1991). Ha sido la serie sobre Instrumenta Inscripta Latina la primera en poner verdaderamente en valor dicha documentación (Hainzmann y Borhidi, 1991 y Hainzmann y Wedenig, 2008). Curiosamente, los textos escritos en griego sobre vidrio sí llamaron tempranamente la atención de la investigación epigráfica peninsular, como demuestran algunos intentos de recopilación realizados bien sin ánimo de exhaustividad (Almagro, 1952: 185-189), bien sin individualizar las marcas sobre vidrio en el contexto de las evidencias epigráficas griegas y, por tanto, con un carácter global (De Hoz GarcíaBellido, 2014: 184 y 213-214). Tampoco la generalización de las bases de datos epigráficas online ha mejorado la situación. Por ejemplo, para las provincias hispanas, la opción «Material/vitrum» del Epigraphische Datenbank Clauss-Slaby (http://manfredclauss.de/) no arroja resultados para muchas de las provincias del Imperio — desde luego, no los arroja 
para ninguna de las provincias hispanas-y la opción «Tipo de objeto/vidrio» del buscador de Hispania Epigraphica Online (http://eda-bea.es/) solo proporciona tres resultados, mientras que recientes inventarios (Salido y Madariaga, 2019) demuestran la presencia de un elenco mucho más generoso de testimonios, que, además, tuvieron una notable pervivencia técnica a través del tiempo (Taylor, 1979: 6-15, n. ${ }^{\text {os }}$ 9, 10, 17 y 23-28).

Cualquier tipología que sobre este tipo de marcas quiera hacerse, debe, necesariamente, distinguir entre los textos grabados sobre vidrio a posteriori y aquéllos realizados a molde en el marco del proceso de fabricación del mismo, como es el que nos ocupa y que, además, está siempre en proceso de transformación e incremento ante nuevos hallazgos como el que aquí se presenta. A título de ejemplo, en la Tarraconense hispana se han publicado recientemente ejemplos de cada una de esas dos categorías, con abundante bibliografía al respecto, que complementa la que aquí se citará. Al primer tipo pertenece la inscripción latina grabada sobre un vidrio de Veranes (Asturias) (Salido y Madariaga, 2018, con repertorio de casos utilísimo en Salido y Madariaga, 2019: 151-158) o varios fragmentos, con inscripción también latina a molde, vinculados a la ciudad de Clunia (Coruña del Conde, Burgos), también muy recientemente dados a conocer (Del Hoyo et al., 2019) y que se añaden al hermoso crismón con letra griega publicado en su día por Palol (1991).

Teniendo en cuenta estos sesgos de la atención bibliográfica a la cuestión y la constante aparición, que no siempre publicación, de nuevos ejemplares, y recopilando las distintas variantes atestiguadas tanto en textos griegos como en latinos, varios son los mensajes que pueden constatarse y sobre los que podría trazarse la siguiente tipología, acaso complementaria de otras conocidas (Györ, 2008: 36-37 y, también, con una tipología más centrada en el responsable del mensaje que en el contenido del mismo, en Sternini, 1994, que distingue entre "bolli pertinente a vetrai», "bolli relativi alla casa imperiale» y «bolli di vetrai o di committenti») y, como se verá, semejante, también, en temas a los que resultan habituales en los grafitos sobre producciones cerámicas, tanto ante cocturam como post cocturam (Lassère, 2005: 440-448), a saber:

1. Inscripciones de carácter identificativo. Formarían parte de este grupo rótulos impresos mediante la técnica del soplado a molde, preferiblemente presentes en cuencos de aspecto invasado, en los que los textos describen, normalmente, personajes de escenas gladiatorias o circenses, cuya representación se desarrolla rodeando el vaso, bien en un solo registro - uno para el texto, a modo de orla, y otro para la escena- bien en varios, intercalando imágenes y texto, configurando viñetas. La técnica narrativa está bien atestiguada en la epigrafía musivaria romana (Corbier, 2005: 105-128) y constituye un género en sí mismo en la iconografía clásica y postclásica (Sparrow, 1969). A este grupo pertenece, por ejemplo, la extraordinariamente bien difundida serie de cuencos con alusión a los aurigas Antiocus, Crescens, Eutyche, Hierax, Musclosus, Pius u Olympus (RIB 2-2, 2419, 5 de Venta Icenorum, en Britannia; AE, 1946, 235 de ThielleWavre, en Germania Superior; sobre ellos: Collingwood y Wright, 1991: 86-92, además de Pfahl, 2012: 210-227 y Taf. 98-100 y Rütti, 1987: 122-123; CIL, XIII, 10025, 176 de 
Haedui, en la Gallia Lugdunensis; HEp 11, 322 de Complutum e IRC V, 180 de Emporiae, en la Tarraconense hispana, este último con estudio monográfico en Darder (1988) o a los gladiadores y atletas Calamus, Columbus, Damocrates, Hermes, Petraites, Prudens o Pyramis (RIB 2-2, 2419, 26 de Londinium, en Britannia; CIL, XIII, 10025, 172 y 180 de Couvin y de Augusta Treuerorum, en la Gallia Belgica; CIL, XIII, 10025, 178 y AE, 1998, 938b de Chavagnes-en-Paillers, en Aquitania), cuyos nombres (Collingwood y Wright, 1991: 93-96; Pfahl, 2012: 218; Moirin, 2003: 216-217, y en Fontaine y Foy, 2015: 97-111) acompañan las escenas de los ludi en que participaron - a veces con hermosas recreaciones arquitectónicas de sus escenarios (RIB 2-2, 2419, 1 de Camulodunum, en Britannia) - , siguiendo una temática bien conocida también para algunas producciones cerámicas (Mayer, 1998, con bibliografía y paralelos). En ocasiones, estos vasos en vidrio soplado a molde con tales tituli han sido catalogados como sports cups (Price y Cottam, 1998: 63-64 y Whitehouse, 1997: 15, o Grose, 2017: 103) y, sabemos, alcanzaron una notable difusión geográfica (Fremersdorf, 1959: 52-54).

2. Inscripciones de carácter descriptivo. Están, en su mayor parte, grabadas y participan del espíritu de la categoría anterior, ayudando básicamente a descodificar de manera adecuada ciertas representaciones gráficas - a diferencia de lo que sucede en el tipo 1, en éste, normalmente, se representan edificios y construcciones - incorporadas a la decoración de unos vasos normalmente difundidos como souvenirs por sus portadores. Los ejemplares, muy bien atestiguados por el Mediterráneo, de los vasos de Puteoli y de Baiae, así como el de Populonia, con hermosas representaciones de sus puertos, constituyen los mejores paradigmas de esta categoría (CIL, XI, 6710, 18 y CIL, XV, 7008 en Italia, y AE, 2005, 763 de Augusta Emerita, en Lusitania, entre otras, este último con estudio monográfico en Bejarano [2005]). Sobre éstos puede verse, además de Picard (1959), la recopilación de Painter (1975), además de los ejemplos de Whitehouse (1997: vol. I, 270-272, n. ${ }^{\circ} 458$ ).

3. Inscripciones de propiedad. Parece que deben interpretarse así algunas marcas muy exclusivas y poco atestiguadas de naturaleza onomástica, como la de C. Caesi Bucaddi en una copa de aspecto antropomorfo y con representación negroide - un género habitual en época altoimperial- de Londinium (RIB 2-2, 2419, 36).

4. Inscripciones de tipo sapiencial o de exhortación, a veces también llamadas «dedicatorias» (Cooley, 2012: 109-111). A la manera de los mensajes que aparecen en muchos de los grafitos post cocturam de la cerámica (véase repertorio en Abascal y Cebrián, 2007), el vidrio incorporó ocasionalmente rótulos con lemas diversos, grabados sobre él y contemplados ya en el molde en el cual se soplaba el recipiente o en piezas de tipo gold glass — estas últimas generalmente tardoantiguas y algunas de ellas con un carácter narrativo semejante al mostrado en el tipo primero (Lutraan, 2006) - , normalmente alusivos a deseos de salud y prosperidad para el portador o

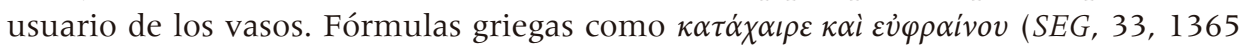
de Serra, en Aegpytus; SEG, 51, 1464, 1 de varios lugares de la Gallia Narbonensis y 1485 de Sidi el Hani, en Africa Proconsularis) (Arveiller-Dulong y Nenna, 2005: 194 
y 294, además de Whitehouse, 1997: vol. I, 239-252, n. ${ }^{\text {os }} 828$-845 y de De Hoz

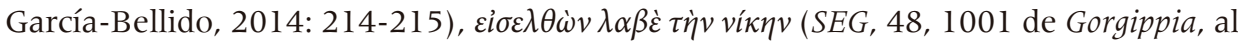

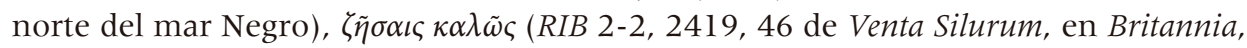

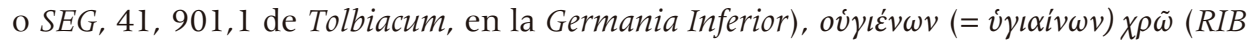
2-2, 2419, 43 de Sussex, Britannia), o latinas como amica bibe (CIL, XIII, 10025, 199 de Durocortorum, en la Gallia Belgica), anni boni (CIL, XV, 7055 de Roma), dulcis uiuas (ICERV 575 de Ossonoba, en Lusitania, o AE, 1997, 171 de Roma), felix pie zesaes cum tuis (AE, 2000, 1123 de Augusta Vindelicorum, en Raetia, o CIL, XIII, 10025, 220 de Bononia, en la Gallia Belgica), semper gaudeatis (AE, 2000, 1215 de Lugio, en Pannonia Inferior), utere felix (CIL, XIII, 10025, 214 de Bononia, en Gallia Belgica, o AE, 1981, 713 de Brigetio, en Pannonia Superior), uita bona fruamur felices (Whitehouse, 2000, además de Whitehouse, 1997: vol. I, 268-270, n. $\left.{ }^{\circ} 457\right)$, uiuas cum tuis pie (AE, 2016, 1135 de Leverkusen; CIL, XIII, 10025, 211 de Viromandis, en la Gallia Belgica, o AE, 1958, 1110 de Wint Hill, en Britannia), uita tibi (CIL, XIII, 10025, 209 de Mogontiacum, en Germania Superior), entre otras, formarían parte de esta categoría (Auth, 1996 y Filippini, 1996, además de Cabart, 2006: 161-176, esp. 170 y Whitehouse, 1997: vol. II, 29, n. ${ }^{\circ}$ 486). 5. Inscripciones de fabricación. Sin duda, la categoría más tempranamente sistematizada en el marco de los estudios epigráficos y, también, la más compleja, generosa y diversa - particularmente en cuanto a las posiciones que los tituli ocupan en el vaso-. Incluye toda una amplísima serie de rótulos — en este caso mayoritariamente a molde- en que o bien figura solo el nombre del productor (uitrarius) en nominativo o en genitivo, o el nombre del productor acompañado de algún verbo alusivo a la realización de la pieza, o fórmulas que indican la naturaleza del negocio en que se ha elaborado el material portante de la marca o, finalmente, siglas y símbolos gráficos abreviando algunas de las posibilidades previas (MacMahon y Price, 2005; Stern, 1999: 458-459, además del clásico trabajo de Foy y Nenna, 2001: 67-68).

Efectivamente, estas inscripciones, ocasionalmente denominadas notae uitrariorum, configuraron ya una categoría específica en la pars VI, dedicada a los uascula uitrea, del Corpus Inscriptionum Latinarum (Dressel, 1899: 871-883), que las ordenó distinguiendo entre las inscripciones ubicadas en las asas (notae impressae in ansulis schyphorum), en los fondos (notae impressae sub fundis ampularum), en las botellas de forma cuadrangular (notae impressae sub fundis uasorum unguentariorum formae quadratae) y sobre fondos de otras formas de vasos (notae impressae sub fundis uasorum uitreorum uarii generis), configurando, también, una categoría específica para los monogrammata iconográficos. Todos estos tipos de marcas aparecen sobre distintas partes de los vasos (para ellas véase Price y Cottam, 1998: 22-29): las asas de apéndice (Whitehouse, 1997: vol. II, 94-97, n. ${ }^{\text {os }}$ 136-141), directamente sobre las paredes (Whitehouse, 1997: vol. II, 63, n. ${ }^{\circ} 76 ; 278-279$, n. $^{\text {os }} 470$ y 485 ) o sobre ellas pero con cartela detallada (Whitehouse, 1997: vol. II, 287-288, n. ${ }^{\text {os }} 482$ y 483; Price, 1991 : 57, Plate XV y 65-67, figs. 7-8, donde también se ofrece un buen repertorio comentado sobre los tipos anteriores con ejemplos), y, por supuesto, como el caso que nos ocupa, 
también sobre la base (algunos ejemplos se recogen en Kisa, 1908: 707, fig. 285, además de en Whitehouse, 1997: vol. II, 18-27, n. ${ }^{\text {os }} 482-491$, así como 85-97, n. ${ }^{\text {os }}$ 567-589, para las inscripciones sapienciales y de fabricación, y 59-66, n. ${ }^{\text {os }} 530-536$, para las inscripciones de tipo narrativo; para detalles sobre este tipo de posiciones nos remitimos a la bibliografía que, en este mismo trabajo, se facilita y, también, a los volúmenes del Corpus des signatures et marques sur verres antiques citados anteriormente).

Figurarían dentro de estas inscripciones de fabricación, por lo tanto, textos como 'Evvíwv

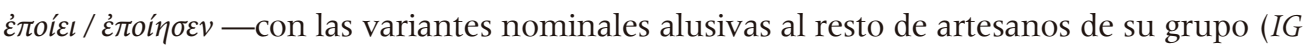
XIV, 2410, 3d de Vercellae, en Italia o IGEP 224a de Tarraco, en Hispania; véase Harden, 1935; Israeli, 1964; Hayes, 1975: 31-47; Stern, 1995: 69-72 y Lightfoot, 2014)—o sus equivalentes latinas Chresimus fecit (RIB 2-2, 2419-101 de Burrium, en Britannia), Felix fecit (CIL, XIII, 10025, 37 de Borbetomagus, en la Germania Superior), Lollianus fecit (AE, 1961, 87a de Sucidava, en la Dacia), Sentia Secunda facit Aq(uileiae) (AE, 1999, 1214b de Lentia, en el Noricum), Amaranthus fecit (AE, 2006, 912 de Nouiomagus, en la Gallia Belgica); fórmulas como ex of(ficina) Laurenti (CIL, XIII, 10025, 144 de Saint-Germain-en-Laye, en la Gallia Narbonensis) o ex of(ficina) Titieni Hyacinthi (CIL, XV, 6997 de Roma); marcas como A(uli) Vol[umin(i)i Ia]nuar(i)i (RIB 2-2, 2419, 84 de Isca, en Britannia), D(ecimi) Veturi Cerdonis (CIL, XV, 803, 4 de Cusercoli, en Italia), Q(uinti) Dani Euhelpisti (AE, 1996, 782 de Segusio, también en Italia), C. Salui Grati (AE, 2016, 1177a de Bratananium, en Raetia), C. Salui Theodor(i) de Augusta Vindelicorum o Quintana, en la Raetia (Pfahl, 2012: 217-218, n. ${ }^{\text {os } 730-732), ~ L . ~ A e m i l i ~ B l a s t i ~(C I L, ~ X I, ~ 6710, ~} 3 a$ de Veleia, en Italia o CIL, XV, 6990b de Roma) (Glöckner, 2008: 133-146), C. Lucreti (CIL, XIII, 10025, 8 de Augusta Treuerorum, en la Gallia Belgica); expresiones como patrimonii (CIL, XIII, 10025, 9c de Bononia, en Italia; AE, 1914, 292 de Deua o AE, 1969-1970, 307 de Cilurnum, ambas en Britannia) o uec(tigal) monopolium p(atrimoni) imp(eratoris) Caes(aris) M(arci) Antonini (AE, 1994, 712b de Verona, Italia). A este grupo también pertenecerían una larga serie de abreviaturas relativas a nomina o cognomina de los fabricantes, tanto en latín como en griego (Taborelli, 1998 y Sternini, 1994, y en los corpora citados anteriormente).

Dentro de esta última categoría (5), el tipo de recipiente de vidrio que, con mayor frecuencia, y también con mayor variedad tipológica, acoge en la península ibérica marcas inscritas (Price, 2006) - particularmente en griego- es el formado por las botellas cuadrangulares del tipo Isings 50 (Isings, 1957: 64-67; también en Facchini, 1999: 285 y en Simon-Hiernard, 2000: 135-153) o los recipientes cuadrados tipo jarra de la forma Isings 62 (Isings, 1957: 81). Ambas tienen una cronología semejante en el valle del Ebro, nunca anteriores a la época de Tiberio (Paz y Ortiz, 2001: 133, además de Allen, 1998; Price y Cottam, 1998: 215 y Lissia, 2000: 58-60; ejemplos en Whitehouse, 1997: vol. I, 182-188, n. ${ }^{\text {os }} 321-329$ y Arveiller-Dulong y Nenna, 2005: 67-70, n. ${ }^{\text {os }}$ 65-78), y presentan cierta eclosión, especialmente, a partir de la época flavia (Paz y Ortiz, 2001: 152), momento en que, parece, ambas formas se generalizarían tanto por Occidente como por Hispania (Caldera de Castro, 1994-1995: 119-121, además de, a título de ejemplo, por la amplitud del repertorio, Alarcão y Étienne, 1976: 157-207; Sánchez de Prado, 2016: 721-723 y Almagro Gorbea y Cereza, 2009: 26-31). El carácter plano del fragmento que nos ocupa, con rehundimiento 


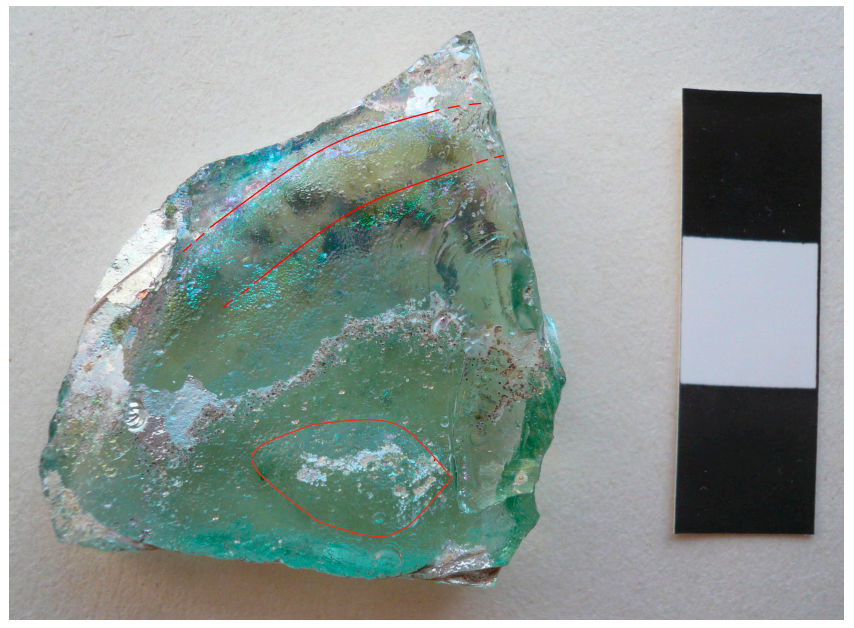

Figura 5. Fondo de botella cuadrangular Isings 50 documentado en Los Bañales, en las excavaciones de las termas, por A. Beltrán Martínez (1972-1979) (foto: Esperanza Ortiz y Juan Á. Paz).

(ejemplos de éstos en Aguilar-Tablada y Sánchez de Prado, 2006: 182-190; Motte y Martin, 2006: 314-315), y las propias dimensiones del mismo $-2,4 \mathrm{~cm}-$ (fig. 4) invitan a pensar que formase parte de un recipiente con una base plana de unos 8,5-9 cm de lado. Estas dimensiones resultan especialmente frecuentes para las botellas Isings 50, que, por otra parte, son las que mejor atestiguadas están en la ciudad romana de Los Bañales — donde, de hecho, consta una base de este tipo con motivo floral (fig. 5) (Ortiz y Paz, 1998: 446 y, con detalle, Ortiz y Paz, 2011: 369-370) — y que, en general, tienen también una presencia más consolidada que la forma Isings 62 tanto en el valle del Ebro (Paz y Ortiz, 2001: 152) como en la Tarraconense (Price, 1981: 359-375), extendiéndose especialmente durante el siglo II d. C. Esa cronología de su difusión encajaría muy bien con la que, desde un punto de vista epigráfico, aportaría el contrastado uso de la grafía $W-\mathrm{y}$ no $\Omega$ - para la letra griega omega, que también parece asentarse entrado el siglo I d. C. y en las primeras décadas del siglo II d. C. Sobre él existen paralelos en marcas sobre vidrio — dentro de alguno de los nombres griegos de uitrarii que se han citado con anterioridad-, así como también en la epigrafía funeraria y monumental de Asia Menor, Grecia y otros lugares del Mediterráneo (Welles, 1938: 355-494 y 573-616, n. ${ }^{\text {os }} 5,114$ y 192; McLean, 2002: 41, para la cronología de ese uso, con ejemplos en $S E G, 56,1322$; TAM V, 1, 616; IG II ${ }^{2} 2050,2055 \ldots$.).

En lo que respecta a la inscripción, la primera conclusión es que nos encontraríamos, muy probablemente, ante un texto que debe leerse $H T I W(\eta \tau \iota \omega)$. Esa lectura retrógrada, condicionada por la perspectiva desde la que la marca se veía a través de la botella, es habitual en los fondos de este tipo de envases. Desde una perspectiva lingüística, la secuencia WITH $(\omega \imath \tau \eta)$ no parece una alternativa probable. A pesar de que la combinación de una omega y una iota sin subscribirse podría darse en un contexto epigráfico, las únicas opciones para esta combinación raramente atestiguada apuntan a palabras de la raíz $\sigma \omega \tau \eta \dot{\rho}$ inscritas con faltas

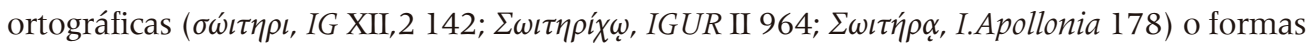




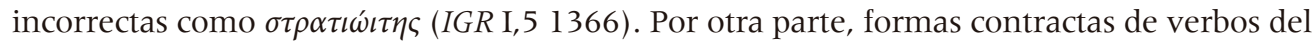
tipo - $\alpha \omega$ en optativo son totalmente descartables en la epigrafía de época imperial. Además, si, como se ha dicho, tenemos en cuenta que en este tipo de soportes lo habitual es la presencia de cognomina - bien en genitivo bien en nominativo- como indicación del nombre del uitrarius, se descartaría también la posibilidad de scriptio continua en el texto, no solo por cuestiones de espacio, como se indica a continuación, sino también porque la terminación $\omega \iota$ únicamente podría referirse a un dativo singular.

A partir de ahí, las propuestas de lectura, teniendo también en cuenta las posibles dimensiones de la botella a la que pertenecería el fragmento que nos ocupa, pueden, más o menos, acotarse convenientemente. Si se trata de una forma Isings 50, cuya base cuadrada, como dijimos, suele medir en torno a los $8-9 \mathrm{~cm}$ de lado, tendríamos espacio suficiente para dar entrada a varias posibilidades onomásticas, mayoritariamente de origen griego, que es lo habitual en este tipo de marcas. Un repaso a los repertorios de nombres atestiguados en vidrios (Trowbridge, 1930: 120-128; Sternini, 1994; Tek, 2003 y Mandruzzato, 2009) nos permite pensar que estaríamos ante un artesano no documentado hasta la fecha; cosa no extraña, dada la notable e ingente variedad de talleres productores —algunos conocidos apenas por una única mención (por ejemplo, Barag, 1987) - y, por supuesto, considerando también la no demasiado generosa publicación de este tipo de evidencias en el contexto de los estudios epigráficos y arqueológicos.

Tras el rastreo en diccionarios, bases de datos (también papirológicas) y la versión digital del Thesaurus Linguae Graecae, destacamos, por su mayor atestiguación en época imperial romana para la secuencia que nos ocupa, las siguientes posibilidades de nom-

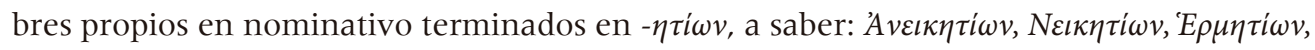
$\Phi \iota \lambda \eta \tau i \omega v, \mathrm{y}$, en menor medida, otros nombres como 'A $\gamma \alpha \pi \eta \tau i \omega v, \Phi \eta \tau i \omega v, K \lambda \eta \tau i \omega v, E \dot{v} \mu \eta \tau i \omega v$,

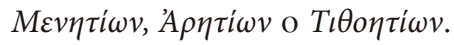

Así, tanto Anicetion como Nicetion y Hermetion constan en la epigrafía romana (Solin, 2003: vol. 1, 353-354 y vol. 2, 833 y 835-836). Por su parte, Philetion —quizás por su mayor dispersión el más apropiado para nuestro texto- está bien atestiguado no solo en la epigrafía de Roma (Solin, 2003: vol. 2, 893), sino también en la Grecia continental y en Asia Menor (Fraser, 2007: vol. IIIA, 450; vol. IV, 343; vol. VA, 448 y vol. VIB, 426), un área esta última de intensa tradición vidriera en época imperial (Degryse et al., 2014: 98 y Grose, 2017: 103117). Esta información onomástica podría orientar a dicho espacio geográfico el origen del productor documentado en nuestro fragmento, si bien la difusión de uitrarii con nombres y marcas griegas por el Mediterráneo nos impide, lamentablemente, ser concluyentes en este sentido (al respecto, véase Harden, 1981: fig. 19). Los dos últimos nombres, Aretion y Tithoetion, estarían principalmente atestiguados en Egipto durante la época imperial (e.g. $S B$ 5, 8961; BGU 2 470; P.Oxy 40, 2937; P.Stras 9, 827; P.Flor 1, 47a; P.Giss.Apoll 37).

La diversidad de posiciones que estas marcas adoptaban en las botellas del tipo de la que nos ha llegado a través de este pequeño fragmento de Los Bañales impide proponer con seguridad un tipo de ordinatio para el texto (véase Lazar, 2006), si bien una línea con el cognomen - a la que pertenecería el fragmento conservado- y otra con el verbo de 


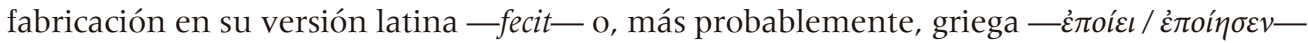
parece la lectio más conveniente, independientemente de que ésta se presentase en dos líneas, como se ha dicho, o, como a veces está atestiguado, se compartimentase partiendo el cognomen y el verbo entre los cuatro lados de la base del recipiente (Nenna, 2006: vol. 3, 257-273, esp. 264, n. ${ }^{\circ}$ PO-IS 52), contorneándola.

Dada la gran difusión que alcanzó el vidrio por el Mediterráneo en época romana y lo extraordinariamente bien valorado que era éste como objeto de consumo (Stern, 2004 y Casagrande, 1998), un hallazgo como el que aquí se presenta —independientemente de que, como es obvio, sea imposible establecer el taller de procedencia- incrementa nuestra información respecto de la apertura comercial de la ciudad romana de Los Bañales, como sabemos, bastante consolidada en el momento en que producciones como éstas comenzaron a generalizarse por la península (Ortiz y Paz, 2019: 795). Esta impresión ya se desprendía del análisis del repertorio de vidrio que, en su día, fuera descrito a partir del recuperado en las excavaciones históricas de Los Bañales de Uncastillo en la década de 1970; apertura comercial que puede confirmarse también con la atestiguación en Los Bañales de un tipo de vidrio, el denominado millefiori, que, recogido históricamente en prospecciones superficiales en Los Bañales -y con varios ejemplares custodiados por J. Planas en una colección particular en Ejea de los Caballeros (Zaragoza)—, está también atestiguado en la ciudad romana, por más que no haya aparecido todavía fragmento alguno en contexto arqueológico (sobre él, véase Oliver, 1968). Este tipo de exóticas producciones sabemos que alcanzó en la vecina Aquitania, y también en la Tarraconense, una notable difusión en el cambio de era (HochuliGysel, 2006: 178-180 y Martínez García, 1999: 17-18), constituyendo un buen fósil director, por su cronología tan acotada (véase, también, Antonaras, 2012: 65-66), y una muestra evidente de la extraordinaria y temprana apertura de la ciudad romana de Los Bañales, en función de su conexión viaria, a los influjos comerciales llegados del exterior (Andreu, 2011 : 40-45). Ese perfil de ciudad abierta se confirma ahora con el nuevo testimonio que aquí se da a conocer y que, pese a su pequeño tamaño, por el interés de su texto en griego, subraya un perfil —el de ciudades de despegue augústeo, con protagonismo territorial y bien comunicadas- que está también presente en ciudades del ámbito galo, aquitano y, obviamente, del propio valle del Ebro (Ortiz, 2001: 25). En todas esas ciudades, además, se ha avanzado más en la caracterización de sus producciones e importaciones en materia de vidrio romano (Moirin, 2003: 221-223 o Foy y Nenna, 2006, además de La Guardia, 1998 y, en relación a este perfil de ciudades, Foy y Nenna, 2006: 106), lo que encaja muy bien con la que parece mostrar, también, este paruum oppidum de Los Bañales entre los siglos I a. C. y II d. C.

\section{Nota}

El presente trabajo se integra en las actividades del Plan de Investigación que, por encargo de la Dirección General de Patrimonio Cultural del Departamento de Educación, Cultura 
y Deporte del Gobierno de Aragón y la dirección de la Fundación Uncastillo, se vienen llevando a cabo en el yacimiento arqueológico romano de Los Bañales (Uncastillo, Zaragoza). El estudio que aquí se presenta se ha visto notablemente enriquecido por las sugerencias aportadas por las arqueólogas M. ${ }^{a}$ Dolores Sánchez de Prado, Belén Madariaga García y Gisela Ripoll, y por el epigrafista Ángel Ventura Villanueva, a quienes queremos mostrar desde aquí gratitud pública. El texto se ha visto también muy enriquecido por las propuestas de los informantes anónimos de la revista Pyrenae. Lógicamente, los errores y omisiones que el texto pueda presentar son responsabilidad única y exclusiva de los firmantes del mismo.

\section{Bibliografía}

ABASCAL, J. M. y CEBRIÁN, R., 2007, Grafitos cerámicos de Segobriga (1997-2006), Lucentum 26, 127-171.

AGUILAR-TABLADA, B. M. ${ }^{a}$ y SÁNCHEZ DE PRADO, M. ${ }^{a}$ D., 2006, Evidencias de un taller de vidrio en la ciudad romana de Augustobriga (Talavera la Vieja, Cáceres), Lucentum 25, 177193.

ALARCÃO, J. y ÉTIENNE, R. (dirs.), 1976, Fouilles de Conimbriga. VI. Céramiques diverses et verres, Boccard, París.

ALLEN, D., 1998, Roman Glass in Britain, Shire Archaeology, Princes Risborough.

ALMAGRO, M., 1952, Las inscripciones ampuritanas griegas, ibéricas y latinas, Instituto Rodrigo Caro de Arqueología, Barcelona.

ALMAGRO GORBEA, M. ${ }^{a}$ J. y CEREZA, E. A., 2009, Vidrios antiguos del Museo Nacional de Artes Decorativas, Real Academia de la Historia, Madrid.

ANDREU, J., 2011, La ciudad romana de Los Bañales (Uncastillo, Zaragoza) en las fuentes históricas, en J. ANDREU (ed.), La ciudad romana de Los Bañales (Uncastillo, Zaragoza): entre la historia, la arqueología y la historiografía, Caesaraugusta 82, Institución Fernando el Católico, Zaragoza, 19-100.
ANDREU, J. (ed.), 2011, La ciudad romana de Los Bañales (Uncastillo, Zaragoza): entre la historia, la arqueología y la historiografía, Caesaraugusta 82, Institución Fernando el Católico, Zaragoza.

ANDREU, J., 2016, Agentes de la monumentalización urbana en una ciudad del norte de la Citerior: Los Bañales de Uncastillo (Zaragoza, España), en A. BOUET (ed.), Monumental! La monumentalisation des villes de l'Aquitaine et de l'Hispanie septentrionale durant le Haut-Empire, Ausonius Éditions, Burdeos, 313-326.

ANDREU, J. y DELAGE, I., 2017, Diuturna atque aeterna ciuitas? Sobre la sostenibilidad de los municipia Latina hispanorromanos a partir de un caso paradigmático: Los Bañales de Uncastillo, en J. ANDREU (ed.), Oppida labentia. Transformaciones, cambios y alteración en las ciudades hispanas entre el siglo II y la tardoantigüedad, Fundación Uncastillo, Uncastillo, 345-374.

ANDREU, J., PEÑALVER, T. y DELAGE, I., 2020, Custodia uiarum publicarum. Signos de debilidad urbana a partir del callejero de una ciudad del interior de la Tarraconense: Los Bañales de Uncastillo (Zaragoza), en J. M. NOGUERA y M. OLCINA (eds.), Ruptura y continuidad: el callejero de la ciudad clásica en el tránsito del Alto Imperio a la Antigüedad Tardía, MARQ, Alicante, 189-196. 
ANTONARAS, A., 2012, Fire and sand. Ancient glass in the Princeton University Art Museum, Yale University Press, New Haven - Londres.

ARVEILLER-DULONG, V. y NENNA, M.-D., 2005, Les verres antiques du Musée du Louvre. II. Vaisselle et contenants du Ier siècle au début du VIIe siècle après J.-C., Musée du Louvre éditions, París.

AUTH, S. H., 1996, Drink may you live! Roman motto glasses in the context of Roman life and death, en Annales du $13^{e}$ Congrès de l'Association Internationale pour l'Histoire du Verre (Pays Bas, 1995), International Association of the History of Glass, Lochem, 103-112.

BARAG, D., 1987, Recent important epigraphic discoveries related to the history of glassmaking in the Roman period, en Annales du $10^{e}$ Congrès de l'Association Internationale pour l'Histoire du Verre (Madrid-Segovia, 1985), Association Internationale pour l'Histoire du Verre, Amsterdam, 109-116.

BEJARANO, A. M. ${ }^{a}, 2005$, Una ampulla de vidrio decorada con la planta topográfica de la ciudad de Puteoli, Mérida Excavaciones Arqueológicas 8, 513 532

CABART, H., 2006, Production et importation de verreries romaines dans l'Est de la France, en D. FOY y M.-D. NENNA (dirs.), Corpus des signatures et marques sur verres antiques. Vol. 1-3, Association Française pour l'Archéologie du Verre, Aix-enProvence, 161-176.

CALDERA DE CASTRO, M. ${ }^{a}$ P., 1994-1995, Los recipientes prismáticos de sección cuadrada y las botellas cilíndricas: una aproximación al método de trabajo de los talleres de vidrio romano del suroeste de Hispania, Anas 7-8, 117-142.

CASAGRANDE, C., 1998, Il vetro in epoca romana: un bene suntuario? Risultato di un'analisi delle fonti giuridico-letterarie latine, en R. LA GUARDIA (ed.), Il vetro dall'antichità all'età contemporanea: aspetii tecnologici, funzionali e commerciali. Atti 2 e giornate nazionali di studio (Milano, 1996), International Association for the History of Glass, Milán, 125-130.

COLLINGWOOD, R. G. y WRIGHT, R. P., 1991, The Roman Inscriptions of Britain. Volume II.
Instrumentum domesticum (personal belongings and the like). Fascicule 2, Alan Sutton, Gloucester.

COOLEY, A., 2012, The Cambridge Manual of Latin Epigraphy, Cambridge University Press, Cambridge, 82-104.

CORBIER, M., 2005, Donner à voir, donner à lire: mémoire et communication dans la Rome ancienne, CNRS, París.

DARDER, M., 1988, Noms d'aurigues i de gladiadors en dos peces de vidre d'Empúries, $\operatorname{ETF}(2)$ 1, 287-300

DEGRYSE, P., CANIO, M. y BOYEN, S., 2014, Primary glass factories around the Mediterranean, en P. DEGRYSE (ed.), Glass Making in the GrecoRoman World, Studies in Archaeological Sciences 4, Leuven University Press, Lovaina, 97-112.

DE HOZ GARCÍA-BELLIDO, M. ${ }^{a}$ P., 2014 , Inscripciones griegas de España y Portugal, Real Academia de la Historia, Madrid.

DEL HOYO, J., SEVILLANO-LÓPEZ, D. y RODRÍGUEZ CEBALLOS, M., 2019, Fragmento epigráfico en vidrio procedente de Clunia, Ficheiro Epigráfico, 185, n. ${ }^{\circ} 689$.

DIANI, M. G. y MANDRUZZATO, L. (dirs.), 2012, Per un corpus dei bolli su vetro in Italia, Comitato Nazionale Italiano dell'Association Internationale pour l'Histoire du Verre (AIHV), Venecia.

DRESSEL, H., 1899, Corpus Inscriptionum Latinarum $X V$. Inscriptiones urbis Romae Latinae. Instrumentum domesticum. Fasc. 1, De Gruyter, Berlín.

FACCHINI, G. M., 1999, Vetri antichi del Museo archeologico al Teatro Romano di Verona e di altre collezioni veronesi, Comitato Nazionale Italiano dell'Association Internationale pour l'Histoire du Verre, Verona.

FILIPPINI, P., 1996, Blown Gold-Sandwich Glasses with Gilt Glass-Trail Inscriptions, en Annales $d u 13^{e}$ Congrès de l'Association Internationale pour l'Histoire $d u$ Verre (Pay Bas, 1995), International Association of the History of Glass, Lochem, 113-128.

FONTAINE, S. y FOY, D., 2015, Mould-blown beakers with figurative scenes: new data on Narbonensis province, en J. BAYLEY, I. 
FREESTONE Y C. JACKSON (eds.), Glass of the Roman World, Oxbow Books, Oxford-Filadelfia, 97-111.

FOY, D. y NENNA, M.-D., 2001, Tout feu, tout sable. Mille ans de verre antique dans le Midi de la France, Musées de Marseille, Marsella.

FOY, D. y NENNA, M.-D. (dirs.), 2006, Corpus des signatures et marques sur verres antiques. Vol. 1-3, Association Française pour l'Archéologie du Verre, Aix-en-Provence.

FOY, D. y NENNA, M.-D., 2006, Productions et importations du verre antique dans la vallée du Rhône et le Midi méditerranéen de la France ( $\mathrm{I}^{\mathrm{er}}-\mathrm{III}^{\mathrm{e}}$ siècles), en D. FOY y M.-D. NENNA (dirs.), Corpus des signatures et marques sur verres antiques. Vol. 1-3, Association Française pour l'Archéologie du Verre, Aix-en-Provence, 227-296.

FRASER, P. M. (ed.), 2007, Lexicon of Greek Personal Names, Clarendon Press, Oxford.

FREMERSDORF, F., 1959, Römische Gläser mit Fadenauflage in Köln: Schalgenfadengläser und Verwandtes, Reykers, Colonia.

GLÖCKNER, G., 2008, Inschriften auf römischen Glasgefäßen aus Österreich, en M. HAINZMANN y R. WEDENIG (eds.), Instrumenta Inscripta Latina. II. Akten des 2. Internationalen Kolloquiums Klagenfurt 2005, Verlag des Geschichtsvereines für Kärnten, Klagenfurt, 133-164.

GROSE, D. F., 2017, The Hellenistic, Roman and Medieval Glass from Cosa, The University of Michigan Press, Ann Arbor.

GYÖR, Z. K., 2008, Inschriften auf Gläser aus der Römerzeit, en M. HAINZMANN y R. WEDENIG (eds.), Instrumenta Inscripta Latina. II. Akten des 2. Internationalen Kolloquiums Klagenfurt 2005, Verlag des Geschichtsvereines für Kärnten, Klagenfurt, 36-37.

HAINZMANN, M. y BORHIDI, A. (eds.), 1991, Instrumenta inscripta Latina: das römische Leben im Spiegel der Kleininschriften, Janus Pannonius Múzeum, Pécs.

HAINZMANN, M. y WEDENIG, R. (eds.), 2008, Instrumenta Inscripta Latina. II. Akten des 2.
Internationalen Kolloquiums Klagenfurt 2005, Verlag des Geschichtsvereines für Kärnten, Klagenfurt.

HARDEN, D. B., 1935, Romano-Syrian Glasses with Mould-blown Inscriptions, The Journal of Roman Studies 25/2, 163-186.

HARDEN, D. B., 1981, Catalogue of Greek and Roman glass in the British Museum. Vol. I. Core- and rod-formed vessels and pendants and Mycenaean cast objects, British Museum Publications, Londres.

HAYES, J. W., 1975, Roman and Pre-Roman Glass in the Royal Ontario Museum. A Catalogue, Royal Ontario Museum, Toronto.

HOCHULI-GYSEL, A., 2006, L'Aquitaine : importations et productions au $\mathrm{I}^{\mathrm{er}}$ siècle av. J.-C. et au I ${ }^{\text {er }}$ siècle ap. J.-C., en D. FOY y M.-D. NENNA (dirs.), Corpus des signatures et marques sur verres antiques. Vol. 1-3, Association Française pour l'Archéologie du Verre, Aix-en-Provence, 177-194.

ISINGS, C., 1957, Roman glass from dated finds, J. B. Wolters, Groningen.

ISRAELI, Y., 1964, Sidonian mold-blown glass vessels in the Museum Haaretz, Journal of Glass Studies 6, 34-41.

KEPPIE, L., 1991, Understanding Roman Inscriptions, Batsford, Londres.

KISA, A. C., 1908, Das Glas im Altertume, Hiersemann, Leipzig.

LA GUARDIA, R. (ed.), 1998, Il vetro dall'antichità all'età contemporanea: aspetii tecnologici, funzionali e commerciali. Atti 2 g giornate nazionali di studio (Milano, 1996), International Association for the History of Glass, Milán.

LASSÈRE, J.-M., 2005, Manuel d'épigraphie romaine, Vol. I, Picard, París.

LAZAR, I., 2006, Base marks on the glass vessels in Croatia: Catalogue, en D. FOY y M.-D. NENNA (dirs.), Corpus des signatures et marques sur verres antiques. Vol. 1-3, Association Française pour l'Archéologie du Verre, Aix-en-Provence, 273-288.

LIGHTFOOT, Ch. S., 2014, Ennion: master of Roman glass, The Metropolitan Museum of Art, Nueva York. 
LISSIA, D., 2000, La collezione dei vetri romani del Museo Archeologico G. A. Sanna di Sassari, Ministero per i Beni e le Attività Culturali, Sassari.

LUTRAAN, K. L., 2006, Late Roman Gold-Glass: Images and Inscriptions, McMaster University, Hamilton.

MACMAHON, A. y PRICE, J., 2005, Glassworking and glassworkers in cities and towns, en Roman working lives and urban living, Oxbow Books, Oxford, 167-190.

MANDRUZZATO, L., 2009, Bottiglie in vetro con marchio da recenti scavi ad Aquileia e Trieste, Quaderni Friulani di Archeologia 19, 159-163.

MARTÍNEZ GARCÍA, A. B., 1999, El vidrio en el campamento romano del Ala II Flavia Hispanorum civium Romanorum en Petavonium (Rosinos de Vidriales, Zamora), Instituto de Estudios Zamoranos, Zamora.

MAYER, M., 1998, Propuesta de lectura para el vaso de los circienses del alfar de La Maja, Kalakorikos 3, 187-192.

MCLEAN, B. H., 2002, An Introduction to Greek Epigraphy of the Hellenistic and Roman Periods: From Alexander the Great to the Reign of Constantine (323 BC-AD 337), University of Michigan Press, Ann Arbor.

MOIRIN, A., 2003, Contacts et échanges au I $^{\text {er }}$ siècle : l'exemple de la Gaule du centre, en D. FOY y M.-D. NENNA (eds.), Échanges et commerce du verre dans le monde antique, Mergoil, Montagnac, 211-226.

MOTTE, S. y MARTIN, S., 2006, L'atelier de verrier antique de la Montée de la Butte à Lyon et ses productions, en D. FOY y M.-D. NENNA (dirs.), Corpus des signatures et marques sur verres antiques. Vol. 1-3, Association Française pour l'Archéologie du Verre, Aix-en-Provence, 303320.

NENNA, M.-D., 2001, Verres de l'Antiquité grécoromaine : cinq ans de publication (1995-1999), Revue Archéologique 32/2, 302-342.

NENNA, M.-D., 2006, Marques en grec, en D. FOY y M.-D. NENNA (dirs.), Corpus des signatures et marques sur verres antiques. Vol. 1-3, Association Française pour l'Archéologie du Verre, Aix-enProvence, 257-273.

OLIVER, A., 1968, Millefiori Glass in Classical Antiquity, Journal of Glass Studies 10, 48-70.

ORTIZ, E., 2001, Vidrios procedentes de la provincia de Zaragoza: el Bajo Imperio Romano. Catálogo: fondos del Museo de Zaragoza, Institución Fernando el Católico, Zaragoza.

ORTIZ, E. y PAZ, J. Á., 1998, El vidrio en los baños romanos, en M. ${ }^{a}$ J. PÉREX, (ed.), Termalismo antiguo. I Congreso Peninsular, Casa de Velázquez, Madrid, 437-451.

ORTIZ, E. y PAZ, J. Á., 2011 , El vidrio romano en Los Bañales (Uncastillo, Zaragoza): revisión preliminar, en J. ANDREU (ed.), La ciudad romana de Los Bañales (Uncastillo, Zaragoza): entre la historia, la arqueología y la historiografía, Caesaraugusta 82, Institución Fernando el Católico, Zaragoza, 355-387.

ORTIZ, E. y PAZ, J. Á., 2019, El vidrio en Hispania romana (218 a. C. - 472 d. C.), en E. H. SÁNCHEZ LÓPEZ y M. BUSTAMANTE (coords.), Arqueología romana en la Península Ibérica, Editorial Universidad de Granada, Granada, 795-809.

OSTROW, S. E., 1979, The topography of Puteoli and Baiae on the eight glass flasks, Puteoli 3, 77-137.

OZCÁRIZ, P., 2009, El instrumentum domesticum y el instrumentum inscriptum, en J. ANDREU (ed.), Fundamentos de Epigrafía Latina, Liceus E-Excellence, Madrid, 533-577.

PAINTER, K. S., 1975, Roman flasks with scenes of Baiae and Puteoli, Journal of Glass Studies 17, 54-67.

PALOL, P., 1991, Un vidrio tallado, con temas cristianos, de Clunia, en Clunia 0: Studia Varia Cluniensia, Diputación Provincial de Burgos, Burgos, 349-354.

PAZ, J. Á. y ORTIZ, E., 2001, El vidrio romano en el valle medio del Ebro (provincia de Zaragoza), en Vidrio romano en España: la revolución del vidrio soplado, Fundación Centro Nacional del Vidrio, La Granja de San Ildefonso, 127-173. 
PEÑALVER, T. y DELAGE, I., 2018, IX Campaña de excavaciones en el yacimiento romano de Los Bañales (Uncastillo, Zaragoza): la Domus del Peristilo, Archivo de Prehistoria Levantina 32, 235-259.

PFAHL, S. F., 2012, Instrumenta Latina et Graeca Inscripta des Limesgebietes von 200 v. Chr. bis 600 n. Chr., Greiner, Weinstadt.

PICARD, Ch., 1959, Pouzzoles et le paysage portuaire, Latomus 18, 23-51.

PRICE, J., 1981, Roman glass in Spain: a catalogue of glass found at the Roman towns of Tarragona, Mérida, Italica and Carmona, with a discussion of the vessel forms from these towns and other Roman sites in Spain, University of Wales, Cardiff.

PRICE, J., 1991, Decorated mould-blown glass tablewares in the first century AD, en M. NEWBY y K. S. PAINTER (eds.), Roman glass. Two centuries of art and invention, The Society of Antiquaires of London, Londres, 57-72.

PRICE, J., 2006, Mould-blown and impressed designs and names on vessels in Spain, en D. FOY y M.-D. NENNA (dirs.), Corpus des signatures et marques sur verres antiques. Vol. 1-3, Association Française pour l'Archéologie du Verre, Aix-en-Provence, 283-320.

PRICE, J. y COTTAM, S., 1998, Roman-British Glass Vessels: a Handbook, Council for British Archaeology, York.

ROMERO, L., 2017, La basílica del foro de Los Bañales (Uncastillo, Zaragoza), Habis 48, 113-132.

ROMERO, L. y ANDREU, J. 2018, El programa escultórico del foro de la ciudad romana de Los Bañales: novedades y aspectos iconográficos, programáticos e históricos, en C. MÁRQUEZ y D. OJEDA (eds.), Escultura romana en Hispania. VIII, Universidad de Córdoba, Córdoba, 365-388.

RÜTTI, B., 1987, Römische Gläser aus Augusta Rauricorum, Augst/Kaiseraugst, Schweiz, en Annales du $10^{e}$ Congrès de l'Association pour l'Histoire du Verre (Madrid-Segovia, 1985), Association
Internationale pour l'Histoire du Verre, Amsterdam, 117-134.

SALIDO, J. y MADARIAGA, B., 2018, Fragmento de botella con inscripción procedente de la villa romana de Veranes (Asturias, España), Journal of Glass Studies 60, 25-39.

SALIDO, J. y MADARIAGA, B., 2019, Los vidrios con inscripción grabada en Hispania: contexto, tipología y significado, Pyrenae 50/1, 149-169.

SÁNCHEZ DE PRADO, M. ${ }^{a}$ D., 2016, El vidrio romano en el conuentus Carthaginiensis. Comercio y producción. Tomo II, Universitat d'Alacant, Alicante.

SIMON-HIERNARD, D., 2000, Verres d'époque romaine. Collection des musées de Poitiers, Musées de la ville de Poitiers et de la Société des Antiquaires de l'Ouest, Poitiers.

SOLIN, H., 2003, Die griechischen Personennamen in Rom, De Gruyter, Berlín.

SPARROW, J. H. A., 1969, Visible Words: a Study of Inscriptions in and as Books and Works of Art, Cambridge University Press, Cambridge.

STERN, E. M., 1995, The Toledo Museum of Art. Roman mold-blown glass. The first through sixth centuries, L'Erma di Bretschneider, Roma.

STERN, E. M., 1999, Roman glassblowing in a cultural context, American Journal of Archaeology 103/3, 441-484.

STERN, E. M., 2004, I vetrai dell'antica Roma, en M. BERETTA (ed.), Vitrum. Il vetro fra arte e scienza nel mondo romano, Giunti, Florencia, 37-59.

STERNINI, M., 1993, I vetri, en W. V. HARRIS (ed.), The inscribed economy. Production and distribution in the Roman Empire in the light of instrumentum domesticum, University of Michigan, Ann Arbor, 81-94.

STERNINI, M., 1994, Bottiglie in vetro con bolli greci, en Epigrafia della produzione e della distribuzione, École Française de Rome, Roma, 567-574.

TABORELLI, L., 1998, Riflessioni sul caso di un bollo vitreo con tria nomina forse ridotti a sigla, Athenaeum 86/1, 285-289. 
TABORELLI, L., 2006, Conteneurs, contenus et marques : problématiques et essai d'interprétation, en D. FOY y M.-D. NENNA (dirs.), Corpus des signatures et marques sur verres antiques. Vol. 1-3, Association Française pour l'Archéologie du Verre, Aix-en-Provence, 9-16.

TAYLOR, F. A., 1979, Glass Exhibition: Ashmolean Museum Oxford, Ashmolean Museum, Oxford.

TEK, A. T., 2003, Prismatic glass bottles with Greek inscriptions from Arycanda in Lycia, en Annales du $15^{e}$ Congrès de l'Association Internationale pour l'Histoire du Verre (New York - Corning, 2001), Association Internationale pour l'Histoire du Verre, Nottingham, 82-87.

TROWBRIDGE, M. L., 1930, Philological studies on ancient glass, University of Illinois, Illinois.
URIBE, P., 2015, La arquitectura doméstica urbana romana en el valle medio del Ebro (siglos II a. C. - III d. C.), Ausonius Éditions, Burdeos.

VIGIL, M., 1969, El vidrio en el mundo antiguo, Instituto Español de Arqueología, Madrid.

WELLES, CH. B., 1938, Inscriptions, en C. H. KRAELING (dir.), Gerasa: City of the Decapolis, American Schools of Oriental Research, New Haven, 355-616.

WHITEHOUSE, D., 1997, Roman Glass in The Corning Museum of Glass. Volume I and volume II, The Corning Museum of Glass, Corning-Nueva York.

WHITEHOUSE, D., 2000, Vasetto con scene di caccia, en S. ENSOLI y E. LA ROCCA (eds.), Aurea Roma. Dalla città pagana alla città cristiana, L'Erma di Bretschneider, Roma, 629-630, n. ${ }^{\circ} 334$. 